\title{
BactQuant: An enhanced broad-coverage bacterial quantitative real-time PCR assay
}

Cindy M Liu², Maliha Aziz ${ }^{1}$, Sergey Kachur ${ }^{1,5}$, Po-Ren Hsueh ${ }^{3}$, Yu-Tsung Huang ${ }^{4}$, Paul Keim ${ }^{1,2}$ and Lance B Price ${ }^{1 *}$

\begin{abstract}
Background: Bacterial load quantification is a critical component of bacterial community analysis, but a culture-independent method capable of detecting and quantifying diverse bacteria is needed. Based on our analysis of a diverse collection of $16 \mathrm{~S}$ rRNA gene sequences, we designed a broad-coverage quantitative real-time PCR (qPCR) assay-BactQuant—for quantifying $16 \mathrm{~S}$ rRNA gene copy number and estimating bacterial load. We further utilized in silico evaluation to complement laboratory-based QPCR characterization to validate BactQuant.

Methods: The aligned core set of 4,938 16 S rRNA gene sequences in the Greengenes database were analyzed for assay design. Cloned plasmid standards were generated and quantified using a qPCR-based approach. Coverage analysis was performed computationally using $>670,000$ sequences and further evaluated following the Minimum Information for Publication of Quantitative Real-Time PCR Experiments (MIQE) guidelines.

Results: A bacterial TaqMan ${ }^{\circledR}$ qPCR assay targeting a 466 bp region in V3-V4 was designed. Coverage analysis showed that $91 \%$ of the phyla, $96 \%$ of the genera, and $>80 \%$ of the 89,537 species analyzed contained at least one perfect sequence match to the BactQuant assay. Of the 106 bacterial species evaluated, amplification efficiencies ranged from 81 to $120 \%$, with $r^{2}$-value of $>0.99$, including species with sequence mismatches. Inter-and intra-run coefficient of variance was $<3 \%$ and $<16 \%$ for $\mathrm{Ct}$ and copy number, respectively.

Conclusions: The BactQuant assay offers significantly broader coverage than a previously reported universal bacterial quantification assay BactQuant in vitro performance was better than the in silico predictions.
\end{abstract}

\section{Background}

Scientists today are studying bacterial communities from diverse habitats, hosts, and health conditions based on the $16 \mathrm{~S}$ rRNA gene [1,2]. To date, most studies have focused on qualitative characterization based on the relative abundances of community bacterial groups [3-5]; however, quantitative characterization-i.e., measurement of the total bacterial load-provides valuable and complementary information when combined with these qualitative data [6]. Traditional culture-based approaches for quantifying bacterial load are inherently limited for assessing the complex bacterial communities that exist in many clinical and environmental samples. Likewise, standard culture-based methods are ineffective for quantifying many fastidious and uncultivable bacterial species [7].

\footnotetext{
* Correspondence: Iprice@tgen.org

'Division of Pathogen Genomics, Translational Genomics Research Institute, 3051 W. Shamrell Blvd., Suite 106, Flagstaff, AZ 86001 USA

Full list of author information is available at the end of the article
}

Among culture-independent approaches, quantitative real-time PCR (qPCR) is currently best suited for measuring bacterial load, because of its intrinsic quantitative capability, ease of use, and flexibility in assay design $[8,9]$. Using the qPCR platform, we can design an assay capable of concurrently detecting and quantifying all unique bacteria that constitutes a complex community. Furthermore, by utilizing $16 \mathrm{~S}$ rRNA gene as the target of a broad coverage qPCR assay, results from the qPCR evaluation can be easily combined with $16 \mathrm{~S}$ rRNA genebased qualitative characterization to fully describe the community of interest.

In the current paper, we present our design and validation of a broad-coverage quantitative real-time PCR assay-BactQuant-for quantifying $16 \mathrm{~S}$ rRNA gene copy number and estimating bacterial load. To accomplish this, we have employed a novel nucleotide distributionbased approach to effectively summarize a large $16 \mathrm{~S}$ rRNA gene sequence dataset for qPCR assay design. We further addressed a general limitation of the $\mathrm{qPCR}$

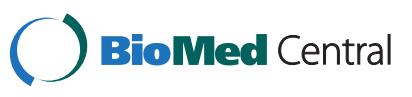

(c) 2012 Liu et al.; licensee BioMed Central Ltd. This is an Open Access article distributed under the terms of the Creative Commons Attribution License (http://creativecommons.org/licenses/by/2.0), which permits unrestricted use, distribution, and reproduction in any medium, provided the original work is properly cited. 
platform-the normalization of in-run quantitative standards using fluorimetric or spectrometric methods-by developing an alternative qPCR-based method for quantifying plasmid standards. Lastly, we have complemented standard qPCR assay validation following MIQE guideline [10] with extensive in silico analysis using $>670,000$ 16 S rRNA gene sequences from the Ribosomal Database Project [11].

\section{Methods}

\section{Design of 16 S rRNA gene quantitative real-time PCR} assay

Pre-aligned $16 \mathrm{~S}$ rRNA gene sequences $(\mathrm{n}=4,938)$ were downloaded from the core set of the Greengenes database [12]. The alignment was analyzed to generate an output of nucleotide distribution-i.e., the summary of allele frequency at each nucleotide position in the $16 \mathrm{~S}$ rRNA gene multiple sequence alignment file-and diversity score using a 3\% gap-filter setting and the Simpson's Diversity Index, respectively.

Assay Design The nucleotide distribution was examined to identify a conserved 500 bp region for assay design. In designing the assays, we applied the following rules: 1) primer sequences cannot have more than three degenerate bases and 2) the probe sequence cannot have any degenerate bases. The primer $\mathrm{T}_{\mathrm{m}}$ was calculated using salt adjusted calculation from the online tool OligoCalc [13] and the probe $T_{m}$ was calculated using the Primer Probe Test Tool for TaqMan ${ }^{\circledR}$ MGB quantification from the Primer Express ${ }^{\circledR}$ Software for Real-Time PCR version 3.0 (Applied Biosystems, Carlsbad, CA, USA) (Table 1).

\section{Computational analysis of assay specificity and coverage}

A. Specificity analysis. Specificity check was performed in GenBank using megablast against human, mouse, and fungal sequences from the nucleotide collection (nr/nt) [14].

B. Collection and identification of bacterial $16 \mathrm{~S}$ rRNA gene sequence eligible for in silico coverage analysis. All $16 \mathrm{~S}$ rRNA gene sequence data used in the in silico coverage analysis were downloaded from the Ribosomal Database Project (RDP) Release 10 Update 20 [11]. Briefly, all bacterial 16 S rRNA gene sequences that were of "Good" quality and had a length of $1200 \mathrm{bp}$ or greater were extracted from RDP, along with taxonomic metadata and sequence IDs. Additionally, the Escherichia coli position data was kindly provided by staff at the RDP. The downloaded sequences were filtered based on E. coli position. Only sequences with data present in the qPCR assay amplicon of interest were considered to be eligible for sequence matching for the particular qPCR assay. Numerical and taxonomic coverage analysis was performed for the BactQuant assay and a published qPCR assay [15] by developing a web service for the RDP Probe Match Tool for sequence matching.

C. Overview of sequence matching analysis for determining assay coverage. All sequence matching for the in silico coverage analysis was performed using two conditions: a) perfect match of full-length primer and probe sequences and $b$ ) perfect match of full-length probe sequence and the last 8 nucleotides of primer sequences at the 3' end. For each sequence matching condition, the in silico coverage analysis was performed at three taxonomic levels: phylum, genus, and species, as well as for all sequences eligible for sequence matching. The remaining taxonomic levels were omitted due to the large amounts of missing and inconsistent data. Details of in silico coverage analyses are as follows:

D. Numerical coverage analysis. At each analysis level, unique operational taxonomic unit (OTU), i.e., each unique taxonomic group ranging from unique phyla to unique species, containing at least one sequence that is a sequence match (i.e., "match") for all three components of the assay of interest were identified using the following requirement: [Forward Primer Perfect Match](union)[Reverse Primer Perfect Match](union)[Probe Perfect Match]. The in silico coverage analysis was performed in a stepwise fashion, beginning with all eligible sequences, then proceeding to analysis at the species-, genus-, and phylum-level. At each step, the taxonomic identification of each sequence was generated by concatenation of relevant taxonomic data (e.g., for species-level analysis, a unique taxonomic identification consisting of concatenated PhylumGenus-species name was considered as one unique species). The sequence IDs were used in lieu of a

Table 1 Primer and probe sequences of BactQuant, the new 16 S rRNA gene-based quantitative real-time PCR (bold letters denotes degenerate base)

\begin{tabular}{llc}
\hline BactQuant & & Tm ('C) \\
\hline Forward Primer & 5'-CCTACGGGDGGCWGCA-3' & $55.9-58.4$ \\
Reverse Primer & 5'- GGACTACHVGGGTMTCTAATC -3' region \\
Probe & (6FAM) 5'-CAGCAGCCGCGGTA-3' (MGBNFQ) & $57.5-63.3$ \\
\hline
\end{tabular}


taxonomic identification for the first analysis step, which included all eligible sequences. The stepwise numerical coverage analysis was performed as follows: all eligible sequences underwent sequence matching with all three components of the assays of interest using a select matching condition (i.e., the stringent or the relaxed criterion). The sequence IDs of matched sequences were assigned and binned as Assay Perfect Match sequence IDs. For this first analysis step, the numerical coverage was calculated using the total number of sequences with Assay Perfect Match sequence IDs as the numerator and the total number of eligible sequences as the denominator. Next, at the species-level, all sequences assigned as Assay Perfect Match sequence IDs were dereplicated based on the concatenated PhylumGenus-species taxonomic identifications. Specieslevel numerical coverage was then calculated using the total number of dereplicated taxonomic identifications as the numerator. Denominator was calculated using the dereplicated Phylum-Genusspecies taxonomic identifications from all eligible sequences. As a result of the logic of this analysis pipeline, a species (i.e., a group of sequences sharing the same unique Phylum-Genus-species designation) was considered an assay sequence match and thus "covered", when at least one Assay Perfect Match sequence ID was in the species group. The numerical coverage analysis was repeated on the genus-level using the dereplicated Phylum-Genus taxonomic identifications from the Assay Perfect Match sequence IDs bin (numerator) and from all eligible sequences (denominator), and lastly, on the phylum-level using Phylum taxonomic identifications. To facilitate calculation of assay coverage, two ambiguous phyla, "Bacteria Insertia Sedis" and "Unclassified Bacteria" were excluded from the phylum-level analysis. Sequences with genus, species, and strain names containing "unclassified" were included in the numerical coverage analyses due to their high abundance.

E. Taxonomic coverage analysis. The in silico taxonomic coverage analysis was performed to generate a detailed output consisting of the taxonomic identifications that were covered or "uncovered" (i.e., no sequence match) at multiple taxonomic levels. A step-wise approach was again utilized for this analysis, beginning with all eligible sequences, performed as follows: First, the Assay Perfect Match sequence IDs were subtracted from the sequence IDs from all eligible sequences, with the resultant sequences assigned and binned as Assay Non-Perfect Match sequence IDs. Next, on the species-level, the Phylum-Genus-species taxonomic identifications of all eligible sequences was first dereplicated, from which the "covered" species taxonomic identifications were subtracted. Species-level taxonomic coverage was then presented as a list of concatenated taxonomic identification of the covered and uncovered species. This was repeated with the genus- and phylum-level taxonomic identifications for genus- and phylumlevel taxonomic coverage analyses. Output of taxonomic identifications from analysis using all eligible sequences was not presented in this manuscript due to its extensive size but is available in Additional file 1: Figure S1.

F. Assay comparison using results from the in silico analyses. Results from the in silico analyses were summarized for assay comparison as follows: The numerical coverage for the BactQuantand published qPCR assays were calculated at three taxonomic levels, as well as for all eligible sequences using both sequence matching conditions and presented as both the numerator and denominator, and percent covered calculated as the numerator divided by the denominator. This was presented in Table 2. Additional comparison of the taxonomic coverage was performed by superimposing the genus-level numerical coverage of the BactQuant assay for each phylum onto a maximum parsimony phylogenetic tree. Construction of the phylum-level phylogenetic tree was performed using MEGA4 with representative full-length $16 \mathrm{~S}$ rRNA gene sequences from each of the 34 phyla analyzed [16]. In addition, each phylum was annotated as not covered or poorly covered by the published qPCR assay if the phylum was uncovered or if $>50 \%$ of the genera within the phylum were uncovered, respectively. A list of the uncovered genera by phylum for the BactQuant assay was also generated. Comparison results using the stringent and relaxed criterion were presented in Figure 1 and Additional file 2: FigureS1, respectively.

\section{Quantification and normalization of cloned plasmid standards}

Overview To obtain accurately quantified plasmid standards for validation the BactQuant assay, a $10^{9}$ copies $/ \mu \mathrm{l}$ plasmid stock was quantified using a qPCR assay targeting portion of the vector using the second derivative maximum analysis algorithm on the LightCyler platform. The resultant crossing point value (i.e., $\mathrm{Cp}$-value) is used in plasmid normalization. The details are as follows:

Generation of normalized $16 \mathrm{~S}$ rRNA gene plasmid standards Amplification of the full $16 \mathrm{~S}$ rRNA gene was performed using E. coli genomic DNA as the template and $16 \mathrm{~S}$ rRNA gene primers $27 \mathrm{~F}$ and 1492R as 
Table 2 Results from numerical coverage analysis performed by comparing primer and probe sequences from BactQuant and the published qPCR assays against $>670,00016$ S rRNA gene sequences from RDP

\begin{tabular}{|c|c|c|c|}
\hline & BactQuant & Published qPCR Assay & Coverage Improvement \\
\hline \multicolumn{4}{|c|}{ A. Perfect match using full length primers and probe } \\
\hline Phyla & $\begin{array}{l}\mathbf{9 1 . 2} \% \\
(31 / 34)\end{array}$ & $\begin{array}{l}\mathbf{6 1 . 8 \%} \\
(21 / 34)\end{array}$ & $+29.4 \%$ \\
\hline Genus & $\begin{array}{c}\mathbf{9 6 . 2 \%} \\
(1778 / 1849)\end{array}$ & $\begin{array}{c}\mathbf{8 0 . 3} \% \\
(1485 / 1849)\end{array}$ & $+15.8 \%$ \\
\hline Species* & $\begin{array}{c}\mathbf{8 3 . 5 \%} \\
(74725 / 89537)\end{array}$ & $\begin{array}{c}\mathbf{6 6 . 3 \%} \\
(59459 / 89646)\end{array}$ & $+17.2 \%$ \\
\hline All Sequences* & $\begin{array}{c}\mathbf{7 8 . 0 \%} \\
(524118 / 671595)\end{array}$ & $\begin{array}{c}\mathbf{6 0 . 9 \%} \\
(409584 / 672060)\end{array}$ & $+17.1 \%$ \\
\hline \multicolumn{4}{|c|}{ B. Perfect match using 8-nt primers with full length probe } \\
\hline Phyla & $\begin{array}{l}\mathbf{9 1 . 2} \% \\
(31 / 34)\end{array}$ & $\begin{array}{l}\mathbf{6 7 . 7 \%} \\
(23 / 34)\end{array}$ & $+23.5 \%$ \\
\hline Genus & $\begin{array}{c}\mathbf{9 7 . 7} \% \\
(1806 / 1849)\end{array}$ & $\begin{array}{c}\mathbf{8 2 . 1 \%} \\
(1518 / 1849)\end{array}$ & $+15.6 \%$ \\
\hline Species* & $\begin{array}{c}\mathbf{8 9 . 1 \%} \\
(79759 / 89537)\end{array}$ & $\begin{array}{c}\mathbf{7 0 . 9 \%} \\
(63533 / 89646)\end{array}$ & $+18.2 \%$ \\
\hline All Sequences* & $\begin{array}{c}\mathbf{8 4 . 4 \%} \\
(566685 / 671595)\end{array}$ & $\begin{array}{c}\mathbf{6 5 . 6 \%} \\
(441017 / 672060)\end{array}$ & $+18.8 \%$ \\
\hline
\end{tabular}

The in silico analysis was performed using two sequence matching conditions.

*The difference in number of sequences eligible for in silico evaluation is due to the difference in primer lengths and locations of the two assays.

previously described [17]. Visualization of PCR amplicon was performed using gel electrophoresis with SYBR 2\% agarose gel. The resultant PCR amplicons were immediately used as the target gene insert with the $\mathrm{TOPO}^{\circledR} \mathrm{TA}$ Cloning ${ }^{\circledR}$ Kit (with $\mathrm{pCR}^{\circledR} 2.1 \mathrm{TOPO}^{\circledR}$ vector) (Invitrogen Corp., Carlsbad, CA, USA) following the manufacturer's instructions. The resultant propagated cloned plasmids were purified using the QIAprep Spin Miniprep Kit (Qiagen Inc., Valencia, CA, USA). Sequence verification of the purified plasmids containing the $16 \mathrm{~S}$ rRNA gene insert was performed with capillary electrophoresis using BigDye $^{\circledR}$ Terminator v3.1 Cycle Sequencing Kit on the 3130 Genetic Analyzer platform (Applied Biosystems, Carlsbad, CA, USA). Quantification of the cloned plasmids was performed by analyzing three 10-fold dilutions using the vector qPCR assay. Normalization was performed using the dilution factor $2^{\Delta \mathrm{Cp}}$, where $\Delta \mathrm{Cp}=10$ (Cp value of non-normalized cloned plasmids).

\section{Pan-bacterial qPCR assay optimization and initial specificity check}

Assay optimization Using the normalized plasmid standards, different primer and probe titrations were tested on the on the 7900HT Real Time PCR System (Applied Biosystems) and evaluated based on reaction efficiency and assay dynamic range for $10 \mu \mathrm{l}$ and $5 \mu \mathrm{l}$ reaction volumes. For $10 \mu \mathrm{l}$ and $5 \mu \mathrm{l}$ reactions, the optimized conditions included $1 \mu \mathrm{l}$ of template into $9 \mu \mathrm{l}$ and $4 \mu \mathrm{l}$ of reaction mix, respectively, with the final reaction containing $1.8 \mu \mathrm{M}$ of each forward and reverse primer, 225 nM the TaqMan ${ }^{\circledR}$ probe, 1X Platinum ${ }^{\circ}$ Quantitative PCR
SuperMix-UDG w;ROX (Invitrogen Corp.) and molecular-grade water. Irrespective of reaction volume, each experiment included an in-run standard curve $\left(10^{2}-10^{8}\right.$ in 10-fold serial dilutions) and no-template controls performed in triplicate. Amplification and real-time fluorescence detections were performed on the 7900HT Real Time PCR System (Applied Biosystems) using the following PCR conditions: $3 \mathrm{~min}$ at $50^{\circ} \mathrm{C}$ for UNG treatment, $10 \mathrm{~min}$ at $95^{\circ} \mathrm{C}$ for $\mathrm{Taq}$ activation, $15 \mathrm{~s}$ at $95^{\circ} \mathrm{C}$ for denaturation and $1 \mathrm{~min}$ at $60^{\circ} \mathrm{C}$ for annealing and extension $\mathrm{x} 40$ cycles. Cycle threshold value (i.e., Ct value) for each $16 \mathrm{~S}$ qPCR reaction were obtained using a manual Ct threshold of 0.05 and automatic baseline in the Sequence Detection Systems v2.3 software (Applied Biosystems).

Initial specificity check against human and fungal genomic DNA Using the optimized assay condition, the newly designed assay was tested against $1 \mathrm{ng}, 100 \mathrm{pg}$, and $10 \mathrm{pg}$ of human genomic DNA (Promega, Madison, WI, USA), C. albicans genomic DNA (American Type Culture Collection, Manassas, VA, USA), the normalized plasmid standards in triplicate reactions.

\section{Laboratory analysis of assay performance using diverse bacterial genomic DNA}

To assess our assay performance against diverse bacteria, we tested our assay against a diverse collection of bacterial genomic DNA to determine the assay efficiency and correlation coefficients. The details are as follows: 


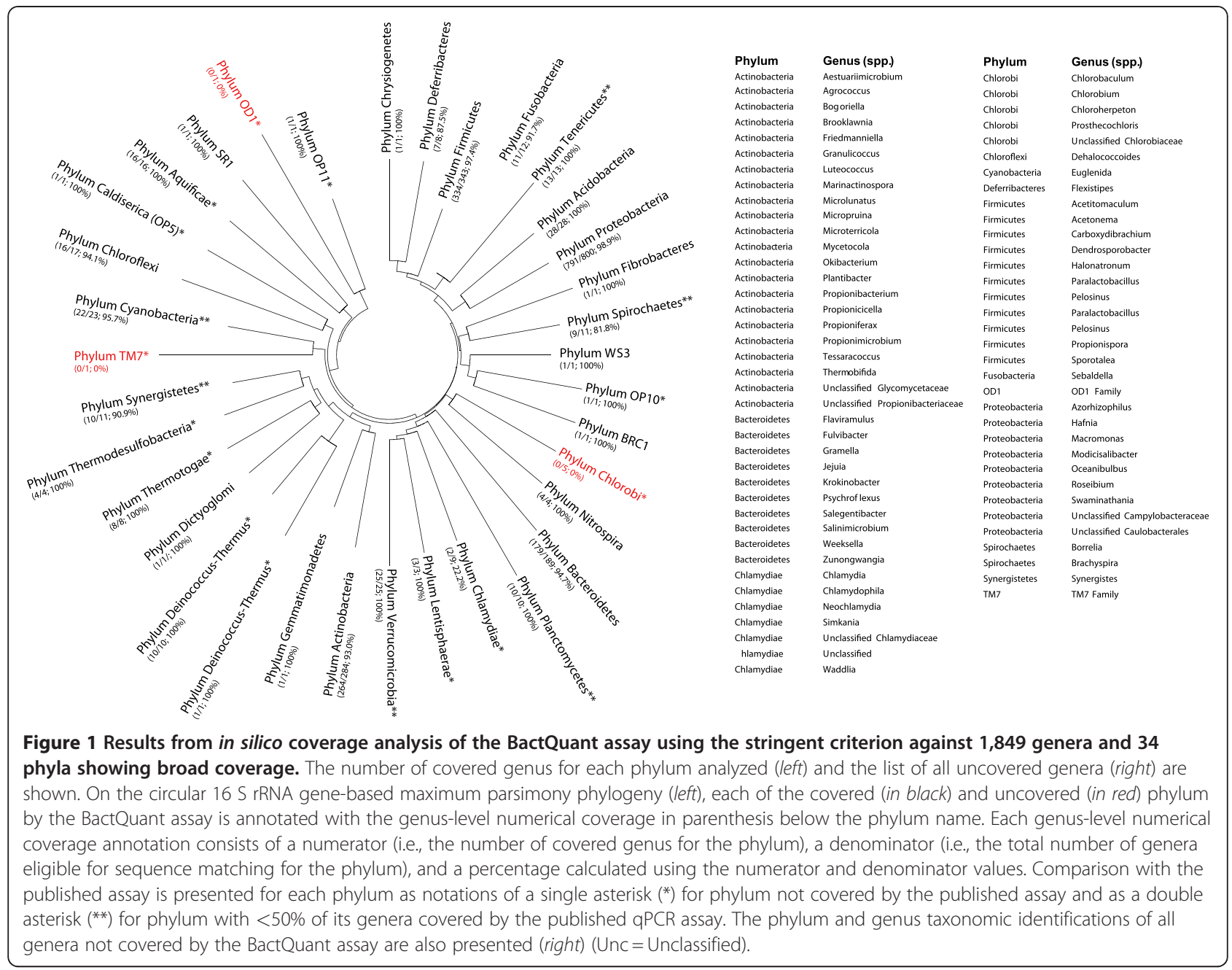

Bacterial strains Arsenophonus nasoniae ATCC 49151, Budvicia aquatica ATCC 51341, Buttiauxella gaviniae ATCC 51604, Cedecea davisae ATCC 33431, Cellvibrio gilvus ATCC13127, Citrobacter freundii ATCC 8090, Clostridium difficile ATCC 9689, Cronobacter aerogenes ATCC 13048, Ewingella americana ATCC 33852, Edwardsiella tarda ATCC 15947, Escherichia vulneris ATCC 33821, Hafnia alvei ATCC 29926, Ewingella americana ATCC 33852, Klebsiella oxytoca ATCC 49131, Kluyvera ascorbata ATCC 33433, Leclericia adecarboxylata ATCC 700325, Leminorella richardii ATCC 33998, Moellerella wisconsensis ATCC 35621, Morganella morganii ATCC 25830, Obesumbacterium proteus ATCC 12841, Pantoea agglomerans ATCC 27155, Photorhabdus asymbiotica ATCC 43950, Plesiomonas shigelloides ATCC 14029, Pragia fontium ATCC 49100, Proteus mirabilis ATCC 29906, Providencia rustigianii ATCC 33673, Pseudomonas aeruginosa ATCC 27853, Pseudomonas andersonii ATCC BAA-267, Pseudomonas anguilliseptica ATCC 33660, Pseudomonas azotofixans ATCC BAA-1049, Pseudomonas fragi ATCC 4973,
Pseudomonas lundensis ATCC 49968, Pseudomonas luteola ATCC 43273, Pseudomonas mendocina ATCC 25411, Pseudomonas monteilii ATCC 700476, Pseudomonas mosselii ATCC BAA-99, Pseudomonas otitidis ATCC BAA-1130, Pseudomonas pseudoalcaligenes ATCC 17440, Psuedomonas putida ATCC 12633, Pseudomonas stutzeri ATCC 17588, Pseudomonas taetrolens ATCC 4683, Rahnella aquatilus ATCC 33071, Raoultella ornithinolytica ATCC 31898, Shigella dysenteriae ATCC 13313, Salmonella enterica ATCC 13076, Serratia liquefaciens ATCC 27592, Tatumella ptyseos ATCC 33301, Trabulsiella guamensis ATCC 49492, Yersinia enterocolitica ATCC 9610, and Yokenella regensburgei ATCC 43001 were obtained from the American Type Culture Collection (Manassas, VA, USA). Bacterial propagation and enrichment were performed under the appropriate condition for each bacterial strain following ATCC recommendations.

Extraction of bacterial genomic DNA Extraction using the enriched broth was performed using ZR Fungal/ 
Bacterial DNA MiniPrepTM (Zymo Research, Irvine, CA, USA) following the manufacturer's instruction. Elution of the purified genomic DNA was performed using $100 \mu \mathrm{l}$ of $1 \mathrm{X}$ TE buffer.

Other sources of bacterial genomic DNA Genomic DNA from Bacteroides fragilis ATCC 25285, Bacteorides ureolyticus GS-15 ATCC 43606, Borrelia burgdorferi strain B31 ATCC 35210, Campylobacter jejuni ATCC 700819, Chlaymydia trachomatis ATCC VR-348B, Chlamydophila pneumoniae ATCC VR-1360D, Fusobacterium nucleatum ATCC 25586, Pectobacterium atrosepticum ATCC BAA672, Pseudomonas syringae ATCC 11355, Streptomyces violaceoruber ATCC BAA-471, Thermus thermophilus ATCC BAA-163, Treponema denticola ATCC 35405, and Vibrio cholera ATCC 39315 were obtained from the American Type Culture Collection. DNA from Mycobacterium avium, subsp. Avium, Mycobacetrium abscessus, Mycobacterium bovis, Mycobacterium chelonae, Mycobacterium gastri, Mycobacterium gordonae, Mycobacterium fortuitum, Mycobacterium kansasii, Mycobacterium marinum, Mycobacterium nonchromogenicum, Mycobacterium phlei, Mycobacterium smegmatis, Mycobacterium vaccae, and Mycobacterium xenopi were kindly provided by $\mathrm{Na}$ tional Taiwan University, Taipei, Taiwan. DNA from clinical isolates of Acinetobacter baumannii, Klebsiella pneumoniae, Burkholderia pseudomallei, Coxiella burnetti, Enterobacter cloacae, Enterococcus faecium, Escherichia coli, Francisella tularensis, Haemophilus influenzae, Legionella pneumophila, Listeria monocytogenes, Moraxella catarrhalis, Neisseria gonorrhoeae, Pseudomonas aeruginosa, Salmonella enterica subsp. enterica serovar gallinarum, Staphylococcus arlettae, Staphylococcus capitis, Staphylococcus cohnii, Staphylococcus epidermidis, Staphylococcus equorum, Staphylococcus hominis, Staphylococcus haemolyticus, Staphylococcus kloosii, Staphylococcus lugdunensis, Staphylococcus saprophyticus, Staphyloccocus xylosus, Streptococcus agalactiae, Streptococcus pneumoniae, and Viridans Streptococcus and were kindly provided by a project supported by NIH/NIAID U01AI066581 at the Translational Genomics Research Institute, Flagstaff, AZ, USA.

Experimental design For sensitivity and efficiency analysis, bacterial genomic DNA from each species was analyzed in three 10-fold serial dilutions in triplicate reactions using the optimized $16 \mathrm{~S}$ qPCR conditions as described above.

Data analysis For each species tested, reaction efficiency and correlation coefficient were calculated using the data from tests against three 10-fold serial dilutions and presented in Table 3. Sequence comparison analysis was performed by aligning the assay primer and probe sequences with $16 \mathrm{~S}$ rRNA gene sequences of the five uncovered species: Borrelia burgdorferi (Genbank Accession No. X98226), Cellvibrio gilvus (Genbank Accession No. GU827555.1), Escherichia vulneris (Genbank Accession No. AF530476), Chlamydia trachomatis (Genbank Accession No. NR025888), and Chlamydophila pneumoniae (Genbank Accession No. CPU68426) in SeqMan ${ }^{\circledR}$. Amplification profile of the five uncovered species were annotated with results from the sequence comparison and presented in Additional file 3: Figure S3A-E.

\section{Laboratory quantitative assay validation using pure plasmid standards and mixed templates}

Assay quantitative validation For the assay quantitative validation, we followed the Minimum Information for publication of Quantitative real-time PCR Experiments, or the MIQE guidelines whenever applicable [10]. The MIQE guidelines were complemented with additional tests to determine assay performance in the presence of background fungal and human genomic DNA. In our experimental design, we included seven template conditions: plasmid standards alone and plasmid standards with $0.5 \mathrm{ng} C$. albicans genomic DNA (ATCC) and with $0.5 \mathrm{ng}, 1 \mathrm{ng}, 5 \mathrm{ng}$, and $10 \mathrm{ng}$ of human genomic DNA per reaction in $10 \mu \mathrm{l}$ reactions and plasmid standards alone in $5 \mu \mathrm{l}$ reactions. For each condition assessed, three qPCR runs were performed to assess reproducibility, or inter-run variability. In each run, three replicate standard curves were tested across the 384-well plate to assess repeatability, or intra-run variability. All reactions were performed in triplicates.

Data analysis Using the data generated, the following assay parameters were calculated: 1 ) inter-run assay coefficient of variation $(\mathrm{CoV})$ for copy number and $\mathrm{Ct}$ value, 2) average intra-run assay $\mathrm{CoV}$ for copy number and $\mathrm{Ct}$. value, 3) assay dynamic range, 4) average reaction efficiency, and 5) correlation coefficient $\left(r^{2}\right.$-value). The limit of detection was not defined for the pure plasmid standards experiments due to variability in reagent contamination. At each plasmid standard concentration, the $\mathrm{Ct}$ standard deviation across all standard curves over three runs was divided by the mean $\mathrm{Ct}$ value across all standard curves over three runs to obtain the inter-run assay $\mathrm{CoV}$. The $\mathrm{CoV}$ from each standard curve from each run (i.e., nine $\mathrm{CoV}$ were used in the calculation for each condition tested) were used to calculate the average and the standard deviation of the intra-run $\mathrm{CoV}$. Linear regression of each standard curve across the full dynamic range was performed to obtain the slope and correlation coefficient values. The slope was used to calculate the reaction efficiency using Efficiency $=10^{(-1 / \text { slope })}-1$. Of note, for each triplicate reaction with $\mathrm{Ct}$ standard deviation 
Table 3 The efficiency and $r^{2}$-value results from laboratory evaluation of the BactQuant assay using genomic DNA from ATCC strains and clinical isolates belonging to 106 unique bacterial species spanning eight bacterial phyla

\begin{tabular}{|c|c|c|}
\hline Species Name & $\begin{array}{l}\text { Reaction } \\
\text { efficiency }\end{array}$ & $\begin{array}{c}r^{2}- \\
\text { value }\end{array}$ \\
\hline Streptomyces violaceoruber & $93 \%$ & $>0.999$ \\
\hline Mycobacterium abscessus & $110 \%$ & $>0.999$ \\
\hline Mycobacterium bovis & $106 \%$ & $>0.996$ \\
\hline Mycobacterium chelonae & $101 \%$ & $>0.999$ \\
\hline Mycobacterium gastri & $104 \%$ & $>0.999$ \\
\hline Mycobacterium gordonae & $104 \%$ & $>0.999$ \\
\hline Mycobacterium fortuitum & $93 \%$ & $>0.999$ \\
\hline Mycobacterium kansasii & $107 \%$ & $>0.999$ \\
\hline Mycobacterium marinum & $110 \%$ & $>0.990$ \\
\hline Mycobacterium nonchromogenicum & $101 \%$ & $>0.999$ \\
\hline Mycobacterium phlei & $104 \%$ & $>0.999$ \\
\hline Mycobacterium smegmatis & $105 \%$ & $>0.999$ \\
\hline Mycobacterium vaccae & $120 \%$ & $>0.999$ \\
\hline Mycobacterium xenopi & $112 \%$ & $>0.999$ \\
\hline Bacteroides ureolyticus & $92 \%$ & $>0.999$ \\
\hline Bacteroides fragilis & $82 \%$ & $>0.993$ \\
\hline Chlamydia trachomatis & N/A & N/A \\
\hline Chlamydophila pneumoniae & N/A & N/A \\
\hline Thermus thermophilus & $97 \%$ & $>0.999$ \\
\hline Clostridium difficile & $88 \%$ & $>0.987$ \\
\hline Listeria monocytogenes & $104 \%$ & $>0.999$ \\
\hline Staphylococcus arlettae & $96 \%$ & $>0.998$ \\
\hline Staphylococcus capitis & $95 \%$ & $>0.993$ \\
\hline Staphylococcus cohnii & $104 \%$ & $>0.999$ \\
\hline Staphylococcus epidermidis & $96 \%$ & $>0.999$ \\
\hline Staphylococcus equorum & $85 \%$ & $>0.997$ \\
\hline Staphylococcus hominis & $108 \%$ & $>0.999$ \\
\hline Staphylococcus haemolyticus & $90-104 \%$ & $>0.999$ \\
\hline Staphylococcus kloosii & $98 \%$ & $>0.999$ \\
\hline Staphylococcus lugdunensis & $94 \%$ & $>0.999$ \\
\hline Staphylococcus saprophyticus & $87-98 \%$ & $>0.999$ \\
\hline Staphylococcus xylosus & $81-100 \%$ & $>0.999$ \\
\hline Streptococcus agalactiae & $98 \%$ & $>0.998$ \\
\hline Streptococcus pneumoniae & $98 \%$ & $>0.999$ \\
\hline Streptococcus viridans & $103 \%$ & $>0.999$ \\
\hline Enterococcus faecium & $91-111 \%$ & $>0.999$ \\
\hline Enterococcus faecalis & $90-100 \%$ & $>0.998$ \\
\hline Fusobacterium nucleatum & $90 \%$ & $>0.999$ \\
\hline Burkholderia pseudomallei & $103 \%$ & $>0.999$ \\
\hline Coxiella burnetti* & $100 \%$ & $>0.998$ \\
\hline
\end{tabular}

Table 3 (Continued)

\begin{tabular}{|c|c|c|}
\hline Francisella tularensis & $100 \%$ & $>0.999$ \\
\hline Legionella pneumophila & $98 \%$ & $>0.999$ \\
\hline Neisseria gonorrhoeae & $95 \%$ & $>0.997$ \\
\hline Pseudomonas aeruginosa & $90-100 \%$ & $>0.999$ \\
\hline Pseudomonas mendocina & $93 \%$ & $>0.999$ \\
\hline Pseudomonas andersonii & $90 \%$ & $>0.999$ \\
\hline Pseudomonas otitidis & $93 \%$ & $>0.999$ \\
\hline Pseudomonas stutzeri & $86 \%$ & $>0.999$ \\
\hline Pseudomonas monteilii & $88 \%$ & $>0.999$ \\
\hline Pseudomonas azotofixans & $84 \%$ & $>0.999$ \\
\hline Pseudomonas mosselii & $92 \%$ & $>0.999$ \\
\hline Pseudomonas luteola & $91 \%$ & $>0.999$ \\
\hline Pseudomonas putida & $90 \%$ & $>0.999$ \\
\hline Pseudomonas fluorescens & $96 \%$ & $>0.999$ \\
\hline Pseudomonas taetrolens & $89 \%$ & $>0.999$ \\
\hline Pseudomonas fragi & $93 \%$ & $>0.999$ \\
\hline Pseudomonas syringae & $95 \%$ & $>0.999$ \\
\hline Pseudomonas pseudoalcaligenes & $93 \%$ & $>0.999$ \\
\hline Pseudomonas lundensis & $93 \%$ & $>0.999$ \\
\hline Pseudomonas anguiliseptica & $93 \%$ & $>0.999$ \\
\hline Cellvibrio gilvus & $92 \%$ & $>0.999$ \\
\hline Acinetobacter baumannii & $100-105 \%$ & $>0.999$ \\
\hline Arsenophonus nasoniae & $87 \%$ & $>0.998$ \\
\hline Budvicia aquatica & $88 \%$ & $>0.999$ \\
\hline Buttiauxella gaviniae & $107 \%$ & $>0.999$ \\
\hline Cedecea davisae & $97 \%$ & $>0.999$ \\
\hline Citrobacter freundii & $95 \%$ & $>0.999$ \\
\hline Cronobacter sakazakii & $96 \%$ & $>0.999$ \\
\hline Edwardsiella tarda & $106 \%$ & $>0.999$ \\
\hline Enterobacter cloacae & $89-111 \%$ & $>0.999$ \\
\hline Enterobacter aerogenes & $107 \%$ & $>0.998$ \\
\hline Escherichia vulneris & $93 \%$ & $>0.999$ \\
\hline Escherichia coli & $91-96 \%$ & $>0.999$ \\
\hline Ewingella americana & $97 \%$ & $>0.999$ \\
\hline Haemophilus influenzae & $91-110 \%$ & $>0.999$ \\
\hline Hafnia alvei & $93 \%$ & $>0.999$ \\
\hline Klebsiella oxytoca & $93 \%$ & $>0.999$ \\
\hline Klebsiella pneumoniae & $95-100 \%$ & $>0.999$ \\
\hline Kluyvera ascorbata & $100 \%$ & $>0.999$ \\
\hline Leclercia adecarboxylata & $93 \%$ & $>0.999$ \\
\hline Leminorella richardii & $94 \%$ & $>0.999$ \\
\hline Moellerella wisconsensis & $93 \%$ & $>0.999$ \\
\hline Moraxella catarrhalis & $91-106 \%$ & $>0.999$ \\
\hline Morganella morganii & $95 \%$ & $>0.999$ \\
\hline Obesumbacterium proteus & $114 \%$ & $>0.994$ \\
\hline
\end{tabular}


Table 3 (Continued)

\begin{tabular}{lcc}
\hline Pantoea agglomerans & $93 \%$ & $>0.999$ \\
Pectobacterium atrosepticum & $90 \%$ & $>0.999$ \\
Photorhabdus asymbiotica & $96 \%$ & $>0.999$ \\
Plesiomonas shigelloides & $93 \%$ & $>0.999$ \\
Pragia fontium & $100 \%$ & $>0.998$ \\
Proteus mirabilis & $98 \%$ & $>0.999$ \\
Providencia rustigianii & $93 \%$ & $>0.999$ \\
Rahnella aquatilis & $92 \%$ & $>0.999$ \\
Raoultella ornithinolytica & $94 \%$ & $>0.999$ \\
Salmonella enterica & $101 \%$ & $>0.999$ \\
Salmonella enterica subsp. enterica serovar & $95 \%$ & $>0.998$ \\
gallinarum & & \\
Serratia liquefaciens & $94 \%$ & $>0.999$ \\
Shigella dysenteriae & $98 \%$ & $>0.999$ \\
Tatumella ptyseos & $101 \%$ & $>0.999$ \\
Trabulsiella guamensis & $95 \%$ & $>0.999$ \\
Yokenella regensburgei & $96 \%$ & $>0.999$ \\
Yersinia enterocolitica & $98 \%$ & $>0.999$ \\
Campylobacter jejuni & $89 \%$ & $>0.999$ \\
Vibrio cholerae & $85 \%$ & $>0.996$ \\
Borrelia burgdorferi & $90 \%$ & $>0.999$ \\
Treponema denticola & $82 \%$ & $>0.999$ \\
\hline *No 16 S rNNA gene sequence available in the Ribosomal Database Project
\end{tabular}

$>0.3$, the triplicates were compared and if a clear outlier was present $(\Delta \mathrm{Ct}>0.3$ from other two replicates), the outlier well was excluded from analysis. Amplification profiles of the seven conditions tested were annotated and presented in Figure 2A-B and Additional file 4: Figure S4A-E. Results from laboratory quantitative validation using all conditions tested were summarized in Table 4. Detailed results of inter- and intra-run coefficient of variation for $\mathrm{Ct}$ value and copy number were presented for all conditions tested in Figure 3 and Additional file 5: Supplemental file $1 \mathrm{~A}-\mathrm{C}$ using scattered plots generated with the vegan package in $R[18,19]$.

Bacteria-to-human ratio calculations Calculations were performed using the following copy number and genome size estimates: the average bacterial $16 \mathrm{~S}$ rRNA gene copy number per genome was estimated to be 3.94 copies as calculated by $\operatorname{rnDB}$ [20] (accessed at http:// ribosome.mmg.msu.edu/rrndb/index.php) and the average human $18 \mathrm{~S}$ rRNA gene copy number per genome was estimated to be 400 copies [21]. The diploid human genome was estimated to be $5,758 \mathrm{Mb}$ [22] or the mass equivalent of $5,758 \mathrm{Mb} /\left(0.978 \times 10^{3} \mathrm{Mb} / \mathrm{pg}\right)=5.887 \mathrm{pg}$ per diploid human genome [23].

\section{Results}

Assay design and initial specificity check

Using our $16 \mathrm{~S}$ rRNA gene nucleotide distribution output, we identified a conserved $500 \mathrm{bp}$ region for assay design. Within this region, we selected three highly conserved sub-regions abutting V3-V4 for the design of a TaqMan ${ }^{\circledR}$ quantitative real-time PCR (qPCR) assay (Additional file 6: Supplemental file 2). Degenerate bases were incorporated strategically in the primer sequence to increase the unique $16 \mathrm{~S}$ rRNA gene sequence types matching the qPCR assay. No degeneracies were permitted in the TaqMan ${ }^{\circledR}$ probe sequence (Table 1). Initial in silico specificity analysis using megablast showed that the probe is a perfect match against human and C. albicans ribosomal DNA, due to its highly conserved nature, but the primers were specific and screening using human and $C$. albicans genomic DNA did not show non-specific amplification.

\section{In silico analysis of assay coverage using $16 \mathrm{~S}$ rRNA gene sequences from 34 bacterial phyla}

Numerical and taxonomic in silico coverage analyses at the phylum, genus, and species levels were performed using $16 \mathrm{~S}$ rRNA gene sequences from the Ribosomal Database Project (RDP) as sequence matching targets. A total of 1,084,903 $16 \mathrm{~S}$ rRNA gene sequences were downloaded from RDP. Of these, 671,595 sequences were determined to be eligible for sequence match comparison based on sequence availability in the E. coli region of the BactQuant assay amplicon. The in silico coverage analyses was performed based on perfect match of full-length primer and probe sequences (hereafter referred to as "stringent criterion") and perfect match with full-length probe sequence and the last 8 nucleotides of primer sequences at the $3^{\prime}$ end (hereafter referred to as "relaxed criterion").

Using the stringent criterion, in silico numerical coverage analysis showed that 31 of the 34 bacterial phyla evaluated were covered by the BactQuant assay. The three uncovered phyla being Candidate Phylum OD1, Candidate Phylum TM7, and Chlorobi (Figure 1). Among most of the 31 covered phyla, more than $90 \%$ of the genera in each phylum were covered by the BactQuant assay. The covered phyla included many that are common in the human microbiome, such as Tenericutes $(13 / 13 ; 100 \%)$, Firmicutes (334/343; 97.4\%), Proteobacteria (791/800; 98.9\%), Bacteroidetes (179/189; 94.7\%), Actinobacteria (264/284; 93.0\%), and Fusobacteria (11/12; 91.7\%). Only three covered phyla had lower than $90 \%$ genus-level coverage, which were Deferribacteres $(7 / 8 ; 87.5 \%)$, Spirochaetes (9/11; 81.8\%), and Chlamydiae $(2 / 9 ; 22.2 \%)$ (Figure 1$)$.

On the genus- and species-levels, 1,778 genera (96.2\%) and 74,725 species $(83.5 \%)$ had at least one perfect match using the stringent criterion. This improved to 1,803 genera $(97.7 \%)$ and 79,759 species $(89.1 \%)$ when the relaxed 


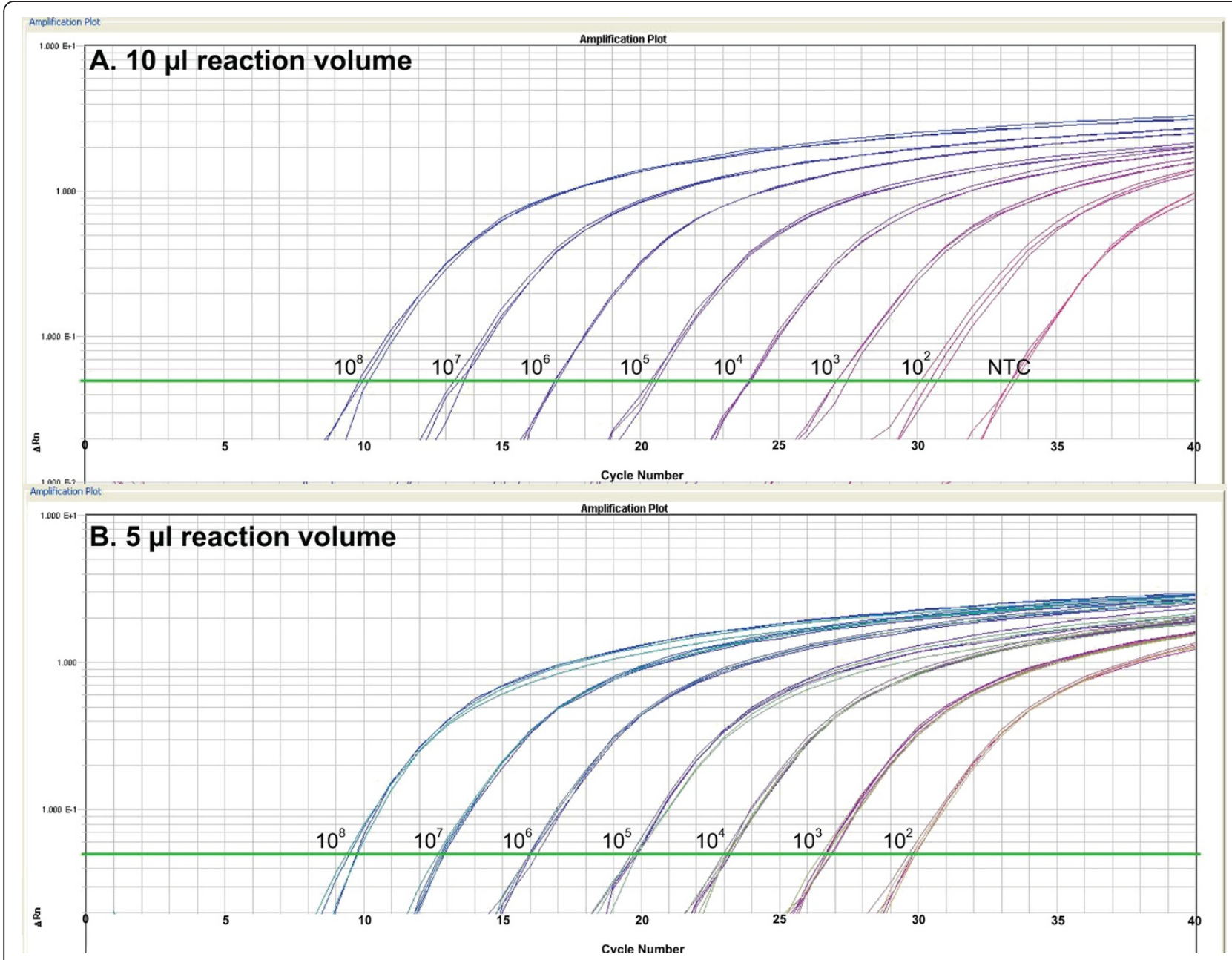

Figure 2 A-B. Standard curve amplification profiles of the BactQuant assay generated from $10 \mu \mathrm{l}$ and $5 \mu \mathrm{l}$ reactions using seven tenfold dilutions and normalized plasmid standards at $10^{9}$ copies/ $\mu$ l. The Ct value of standard curve using $5 \mu$ l reaction volumes (Figure 2B) shows an approximately $1 \mathrm{Ct}$ left shift from the $10 \mu \mathrm{l}$ reaction volumes (Figure 2A). However, the overall amplification profiles are not significantly different between the different reaction volumes over the assay dynamic range of $10^{2}$ copies to $10^{8}$ copies of $16 \mathrm{~S}$ rRNA gene per reaction.

criterion was applied (Table 2, Additional file 2: Figure S1). Using the same relaxed criterion, 566,685 or $84 \%$ of all eligible sequences were perfect matches with the BactQuant assay (Table 2). Detailed taxonomic information on the covered and uncovered OTUs for the BactQuant assay can be found in Additional file 5: Supplemental file 1. Additional file 6: Supplemental file 2.

During our in silico validation, a previously published GPCR assay was identified, which was used as a published reference for comparison [15]. The in silico comparison showed that the BactQuant assay covers more OTUs irrespective of the criterion applied (Table 2, Figure 1, Additional file 2: figure S1). Based on the stringent criterion, the published assay has 10 additional uncovered phyla in comparison to BactQuant; these were: Candidate Phylum OP11, Aquificae, Caldiserica, Thermodesulfoacteria, Thermotogae, Dictyoglomi, Deinococcus-Thermus, Lentisphaerae,
Chlamydiae, and Candidate Phylum OP10 (Figure 1). Applying the relaxed criterion added two phyla, Aquificae and Lentisphaerae, to those covered by the published assay (Additional file 2: figure S1). The genuslevel coverage of the published assay was also low, with fewer than 50\% genus-level coverage in six of its covered phyla. For Cyanobacteria, Planctomycetes, Synergistetes, and Verrucomicrobia, only a single genus was covered by the published assay (Additional file 7: Supplemental file 3). In all, the BactQuant assay covered an additional 288 genera and 16,226 species than the published assay, or the equivalent of $15 \%$ more genera, species, and total unique sequences than the published assay (Table 2). Detailed taxonomic information on the covered and uncovered OTUs for the published qPCR assay can be found in Additional file 7: Supplemental files 3, Additional file 8: Supplemental files 4 . 
Table 4 Laboratory quantitative validation results of the BactQuant assay performed using pure plasmid standards and different mixed templates

\begin{tabular}{lccc}
\hline Templates used & Assay dynamic range & Average reaction efficiency (SD) & $r^{2}$-value \\
\hline Plasmid standards-only (10 $\boldsymbol{\mu l}$ Rxn) & $100-10^{8}$ copies & $102 \%(2 \%)$ & $>0.999$ \\
Plasmid standards-only $\mathbf{5} \boldsymbol{\mu} \mathbf{~ R x n})$ & $100-10^{8}$ copies & $95 \%(1 \%)$ & $>0.999$ \\
Plasmid standards plus $\mathbf{0 . 5}$ ng human gDNA & $100-10^{8}$ copies & $99 \%(4 \%)$ & $>0.994$ \\
Plasmid standards plus $\mathbf{1} \mathbf{~ n g}$ human gDNA & $100-10^{8}$ copies & $96 \%(1 \%)$ & $>0.994$ \\
Plasmid standards plus $\mathbf{5}$ ng human gDNA & $500-10^{8}$ copies & $97 \%(2 \%)$ & $>0.999$ \\
Plasmid standards plus $\mathbf{1 0}$ ng human gDNA & $1000-10^{8}$ copies & $97 \%(1 \%)$ & $>0.999$ \\
Plasmid standards plus $\mathbf{0 . 5}$ ng C. albicans gDNA & $100-10^{8}$ copies &
\end{tabular}

\section{Laboratory analysis of assay performance using diverse} bacterial genomic DNA

Laboratory evaluation of the BactQuant assay showed $100 \%$ sensitivity against 101 species identified as perfect matches from the in silico coverage analysis. The laboratory evaluation was performed using genomic DNA from 106 unique species encompassing eight phyla: Actinobacteria $(n=15)$, Bacteroidetes $(n=2)$, DeinococcusThermus $(n=1)$, Firmicutes $(n=18)$, Fusobacteria $(n=1)$, Proteobacteria $(n=66)$, Chlamydiae $(n=2)$, and Spirochaetes $(n=2)$. Overall, evaluation using genomic DNA from the 101 in silico perfect match species demonstrated $r^{2}$-value of $>0.99$ and amplification efficiencies of 81 to $120 \%$ (Table 3 ).

Laboratory evaluation against the five in silico uncovered species showed variable assay amplification profiles and efficiencies. Of these five species, Chlamydia trachomatis, Chlamydophila pneumoniae, and Cellvibrio gilvus were identified as uncovered irrespective of in silico analysis criterion. However, while C. trachomatis and C. pneumoniae showed strongly inhibited amplification profile, C. gilvus amplified successfully with a $r^{2}$-value of $>0.999$ and an amplification efficiency of $92 \%$ (Additional file 3: Figure S3A-B \& 3E). Two other species, Borrelia burgdorferi and Escherichia vulneris, which were uncovered only when using the stringent criterion, also showed successful amplification with a $r^{2}$-value of $>0.999$ and $90 \%$ and 93\% reaction efficiency, respectively (Additional file 3: Figure S3C-D). Comparison of the assay and bacterial sequences showed that $C$. trachomatis and C. pneumoniae shared a single mismatch in the center of the probe sequence, whereas $C$. gilvus had a mismatch on the 3 ' end of the probe. The mismatch in B. burgdorferi and E. vulneris was a single base difference in $5^{\prime}$ end of the reverse and the forward primer, respectively (Additional file 3: Figure S3A-E). These findings strongly suggest the location of the sequence mismatch is an important determinant of amplification outcome. Furthermore, it supports that the BactQuant assay's coverage in laboratory application is likely greater than determined by the in silico analyses.

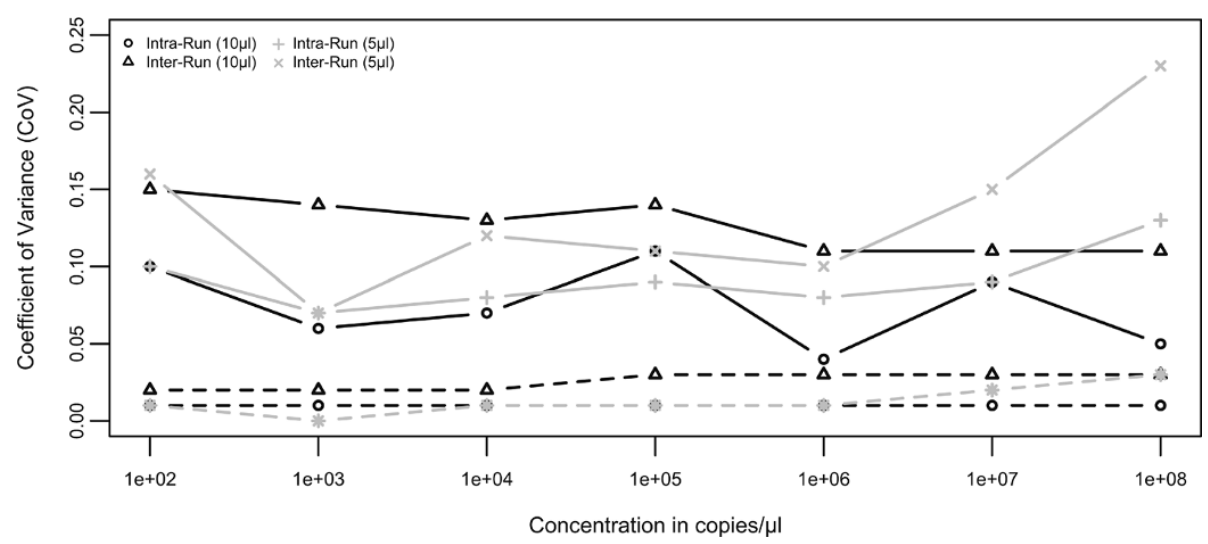

Figure 3 Inter- and intra-run coefficient of variation (CoV) for $10 \mu \mathrm{l}$ and $5 \mu \mathrm{l}$ reactions using seven ten-fold dilutions and normalized plasmid standards at $10^{9}$ copies/ $\mu$ l calculated using data from multiple runs. The data is presented for both copy number (solid line) and Ct value (dashed line). As would be expected, the CoV is higher for copy number than for Ct value and is also higher for inter-run than for intra-run. The Cov for copy number for both reaction volumes was consistently below $15 \%$ until at $10^{7}$ copies for $5 \mu$ reactions. The CoV for $\mathrm{Ct}$ value was consistently below $5 \%$ for both reaction volumes. 


\section{Laboratory quantitative assay validation using pure plasmid standards and mixed templates}

To fully characterize the assay quantitative profile, the BactQuant assay was tested using different reaction volumes and against both pure and mixed templates containing bacterial and human rRNA gene targets. Laboratory evaluation using pure plasmid standards in $10 \mu \mathrm{l}$ and $5 \mu \mathrm{l}$ reaction volumes showed excellent amplification profiles, with an assay dynamic range of $10^{2}-10^{8} \quad 16 \mathrm{~S}$ rRNA gene copies per reaction (Figure $2 \mathrm{~A}-\mathrm{B}$ ). For the $10 \mu \mathrm{l}$ reactions, the inter- and intra-run coefficients of variance $(\mathrm{CoV})$ ranged from $1.58-2.94 \%$ and $0.64-1.25 \%$ for $\mathrm{Ct}$ values and from $10.60-15.36 \%$ and $4.02-10.51 \%$ for copy number, respectively (Figure 3 ). The inter- and intra-run $\mathrm{CoV}$ was comparable for the different reaction volumes, except for the higher $\mathrm{CoV}$ in $5 \mu \mathrm{l}$ reactions containing more than $10^{7}$ plasmid copies (Figure 3 ). This suggests that the $5 \mu \mathrm{l}$ reaction volumes may be better suited for samples with low amounts of bacterial DNA. Establishment of the limit of detection (LOD) for the BactQuant assay using pure plasmid standards was not attempted because it was affected by the level of contaminants in reagents, as previously reported [15,24-28].

Further laboratory evaluations using mixed templates showed that the ratio of bacteria-to-human DNA ratio determined the assay dynamic range of the BactQuant assay (Table 4, Additional file 4: Figure S4A-E, Additional file 5: Additional file 9: Table S1A-C). Experiments using seven tenfold dilutions of plasmid standards with $0.5 \mathrm{ng}$ and $1 \mathrm{ng}$ human gDNA showed that the assay dynamic range was unchanged from pure plasmid standard. However, experiments using $5 \mathrm{ng}$ and $10 \mathrm{ng}$ of human gDNA showed narrower assay dynamic ranges of $500-10^{8}$ and $1000-10^{8}$ $16 \mathrm{~S}$ rRNA gene copies per reaction, respectively. Based on this result, the LODs for $10 \mu \mathrm{l}$ reactions using templates containing $5 \mathrm{ng}$ and $10 \mathrm{ng}$ of human gDNA were estimated to be a bacteria-to-human ribosomal gene copy ratio of 500:339732 and 1000:679464, respectively. This could be further simplified to a bacteria-to-human ribosomal gene copy ratio of 1:679. From a genomic equivalent perspective, the LOD of the BactQuant assay was approximately at a bacteriato-human ratio of 127:849.

\section{Discussion}

We designed and evaluated a new expanded-coverage bacterial quantitative real-time PCR assay targeting the $16 \mathrm{~S}$ rRNA gene. To accomplish this, we curated a set of high-quality $16 \mathrm{~S}$ rRNA gene sequences for assay design and evaluated the coverage of our primers and as a union (rather than as separate entities). In addition, we improved the quantitative capacity of our assay using a cloned plasmid standard. Our computational and laboratory analyses showed that BactQuant had superior in silico taxonomic coverage while retaining favorable in vitro performance. As would be expected, the diverse gene sequences targeted by BactQuant have resulted in variable reaction efficiencies. Nevertheless, laboratory evaluation showed $100 \%$ sensitivity against perfect match species from the in silico analysis.

To allow researchers to determine whether BactQuant covers key organisms in their target community, we provided additional detailed OTU coverage information in the Supplemental Files. We have applied the logic that an OTU was covered if it contained at least one perfect match sequence in the in silico analysis. $16 \mathrm{~S}$ rRNA gene sequences with ambiguous or degenerate bases at the primer and probe sites were considered non-perfect matches, thus making our coverage estimates more conservative. Lastly, although we prohibited the use of a degenerate probe to maximize our assay's quantitative ability, this approach may permit detection of specific taxa such as Chlamydia spp. and Chlamydophila spp.

For most studies, the desired measurement of bacterial load is the number of cells rather than $16 \mathrm{~S}$ rRNA gene copy number; however, the $16 \mathrm{~S}$ rRNA gene copy number varies among bacterial species and even among strains $[29,30]$. The range of copy number is estimated at one to 14, with most non-spore forming species having fewer than 10 copies per genome [20]. We use the average $16 \mathrm{~S}$ rRNA gene copy number per genome from rrnDB in our genomic equivalent estimation, but alternative approaches are possible. This, combined with logarithmic growth of bacteria, suggest that using estimated average copy number could be sufficient.

The in silico analysis was an important component of our validation of BactQuant against diverse bacterial sequence types, even though sequence matching is not a perfect predictor of laboratory performance [31]. Many factors are known to affect reaction efficiency, such as oligonucleotide thermodynamics, the type of PCR master mix used, and the template DNA extraction method. Concentration of background nontarget genomic DNA is another factor that can affect the quantitative parameters rRNA gene-based assays [32]. The interference of background human DNA with BactQuant dynamic range reported in this paper was most likely due to crossreactivity of human DNA with the probe, which targets a region conserved even among eukaryotic organisms, including in the human $18 \mathrm{~S}$ rRNA gene. This may be overcome by using an intercalating reporter dye in place of a fluorescent probe as a $\mathrm{QPCR}$ reporter mechanism; however, the loss of tertiary-level of specificity is a 
potential concern in direct application of an intercalating dye assay to specimens containing high amounts of nontarget DNA.

Exogenous bacterial DNA, particularly from biologically synthesized reagents such as Taq DNA polymerase are a known limitation for analyzing samples with low bacterial load [28,33]. Recently, this issue has received renewed attention due to increased usage of nextgeneration sequencing and the frequent data contamination from exogenous bacterial DNA. Several methods have been evaluated for removing bacterial contaminants from Taq DNA polymerase, including UV irradiation [34,35], DNAse I treatment, and ultrafiltration [36]. The level of $E$. coli contamination in Taq DNA polymerase has been estimated at $10^{2}$ to $10^{5}$ genome equivalents of bacterial DNA per unit of enzyme [28]. This is consistent with the lowest amount of contamination we have observed in our experiments, which were 5 and 10 copies of $16 \mathrm{~S}$ rRNA gene in $5 \mu \mathrm{l}$ and $10 \mu \mathrm{l}$ reactions, respectively. The ubiquity of bacterial DNA also makes the determination of assay specificity challenging.

Our use of qPCR-quantified plasmid standards addressed a major limitation in the preparation of qPCR quantification standards. The conventional approach of quantifying bacterial genomic DNA or plasmid standards necessitates converting mass (i.e., nanograms per $\mu \mathrm{l}$ ) to copy number (i.e., $10^{8}$ copies per $\mu \mathrm{l}$ ) and can introduce substantial error. Thus far, we have also successfully applied BactQuant to a diverse range of clinical specimens, including swab eluents, surgical specimens, and respiratory specimens, but we did not present these findings in this paper. To fully understand the likelihood of false negative results due to interference from human DNA or BactQuant's limit of detection will require additional evaluations.

\section{Conclusion}

In summary, we have developed and evaluated a new broad-coverage qPCR assay-BactQuant-for bacterial detection and quantification that showed concurrently improved assay coverage and favorable quantitative parameters. Laboratory tests showed that in vitro performance was even better than predicted in the in silico analysis; however, our approach of evaluating assay coverage by considering the primer and probe sequences as a single unit is appropriate and necessary. In addition, when employing a copy number estimation method, such as qPCR, the quantification of standards is critical for accurate template quantification. Thus, our approach of quantifying plasmid standards using the intrinsic property of real-time PCR is another important step for any absolute quantification experiments using qPCR.

\section{Additional files} Additional file 1: Figure S1. Figure S1 containing the in silico coverage
analysis using the relaxed criteria.

Additional file 2: Figure S2A-E. Standard curve amplification plots using mixed templates.

Additional file 3: Figure S3A-E. Amplification plots of the non-perfect match targets, including C. trachomatis, C. pneumoniae, C. gilvus, B. burgdorferi, and E. vulneris.

Additional file 4: Figure S4A-E. Coefficient of variance (CoV) distribution across assay dynamic range for mixed templates.

Additional file 5: Supplemental File 1. Detailed results for BactQuant using the stringent criteria.

Additional file 6: Supplemental File 2. Detailed results for BactQuant using the relaxed criteria.

Additional file 7: Supplemental File 3. Detailed results for published assay using the stringent criteria.

Additional file 8: Supplemental File 4. Detailed results from published assay using the relaxed criteria.

Additional file 9: Table S1. Base distribution output used in primer and probe design, with the bolded base signifying the selected base(s) and incorporation of more than one allele at a given nucleotide position was accomplished using degenerate bases. The alignment position

information in the base distribution file contains many gaps as a result from the sequence alignment and differs from the $E$. coli region information from Table 1.

\section{Competing interests}

The authors have declared that no competing interests exist.

\section{Authors' contributions}

CML contributed to the overall study design, the acquisition, analysis, and interpretation of data, and drafting the manuscript, MA contributed to the bioinformatics portion of the study design and its implementation, SK participated in bioinformatics analysis and assay design, PRH and YTH both contributed to the acquisition and interpretation of laboratory data, PK conceived of the study and contributed to the overall study design, LBP contributed to the overall study design and helped to draft the manuscript. All authors read and approved the final manuscript.

\section{Funding}

This work was supported by the National Institutes of Health (R01Al08740901A1, R15DE021194-01), the Department of Defense (W81XWH1010870), and the TGen Foundation. The funders had no role in study design, data collection and analysis, decision to publish, or preparation of the manuscript.

\section{Acknowledgements}

We thank Lindsey Watson (growth and extraction of ATCC isolates and performance of quantitative real-time PCR), Jordan Buchhagen (cloning and sequence verification of cloned standards), Jolene Bowers (generation of the $16 \mathrm{~S}$ rRNA gene plasmid clones), and Tania Contente-Cuomo (performance of quantitative real-time P(R) at Translational Genomics Research Institute for their assistance with different aspects of the laboratory work presented in this manuscript.

\section{Author details}

'Division of Pathogen Genomics, Translational Genomics Research Institute, 3051 W. Shamrell Blvd., Suite 106, Flagstaff, AZ 86001 USA. ${ }^{2}$ Center for Microbial Genetics and Genomics, Applied Research \& Development Building "Northern Arizona University, 1298 S. Knoles Drive, Flagstaff, AZ 86011 USA. ${ }^{3}$ Departments of Laboratory Medicine and Internal Medicine, National Taiwan University Hospital, National Taiwan University College of Medicine, No. 7, Chung-Shan South Road, Taipei, Taiwan. ${ }^{4}$ Department of Internal Medicine, Far Eastern Memorial Hospital, No.21, Nanya S. Rd., New Taipei City, Taiwan. ${ }^{5}$ Current Address: Ross University School of Medicine, 630 US Highway 1, North Brunswick, NJ 08902 USA. 
Received: 28 October 2011 Accepted: 6 March 2012

Published: 17 April 2012

\section{References}

1. Tringe SG, Hugenholtz P: A renaissance for the pioneering $16 \mathrm{~S}$ rRNA gene. Curr Opin Microbiol 2008, 11(5):442-446.

2. Woo PC, Lau SK, Teng JL, Tse H, Yuen KY: Then and now: use of 16S rDNA gene sequencing for bacterial identification and discovery of novel bacteria in clinical microbiology laboratories. Clin Microbiol Infect 2008, 14 (10):908-934.

3. Ravel J, Gajer P, Abdo Z, Schneider GM, Koenig SS, McCulle SL, Karlebach S, Gorle R, Russell J, Tacket CO, et al: Vaginal microbiome of reproductive-age women. Proc Natl Acad Sci U S A 2011, 108(Suppl 1):4680-4687.

4. Grice EA, Kong HH, Conlan S, Deming CB, Davis J, Young AC, Bouffard GG, Blakesley RW, Murray PR, Green ED, et al: Topographical and temporal diversity of the human skin microbiome. Science 2009, 324(5931): 1190-1192.

5. Turnbaugh PJ, Hamady M, Yatsunenko T, Cantarel BL, Duncan A, Ley RE, Sogin $\mathrm{ML}$, Jones WJ, Roe BA, Affourtit JP, et al: A core gut microbiome in obese and lean twins. Nature 2009, 457(7228):480-484

6. Zhang H, Parameswaran P, Badalamenti J, Rittmann BE, Krajmalnik-Brown R Integrating high-throughput pyrosequencing and quantitative real-time PCR to analyze complex microbial communities. Methods Mol Biol 2011. 733:107-128.

7. Zengler K, Toledo G, Rappe M, Elkins J, Mathur EJ, Short JM, Keller M: Cultivating the uncultured. Proc Natl Acad Sci U S A 2002, 99(24): 15681-15686.

8. Hiquchi R, Dollinger G, Walsh PS, Griffith R: Simultaneous amplification and detection of specific DNA sequences. Biotechnology (N Y) 1992, 10(4): 413-417.

9. Bustin SA, Benes V, Nolan T, Pfaffl MW: Quantitative real-time RT-PCR-a perspective. J Mol Endocrinol 2005, 34(3):597-601.

10. Bustin SA, Benes V, Garson JA, Hellemans J, Huggett J, Kubista M, Mueller R, Nolan T, Pfaffl MW, Shipley GL, et al: The MIQE guidelines: minimum information for publication of quantitative real-time PCR experiments. Clin Chem 2009, 55(4):611-622.

11. Cole JR, Wang Q, Cardenas E, Fish J, Chai B, Farris RJ, Kulam-Syed-Mohideen AS, McGarrell DM, Marsh T, Garrity G, et al: The Ribosomal Database Project: improved alignments and new tools for rRNA analysis. Nucleic Acids Res 2009, 37(Database issue):D141-D145

12. DeSantis TZ, Hugenholtz P, Larsen N, Rojas M, Brodie EL, Keller K, Huber T, Dalevi D, Hu P, Andersen GL: Greengenes, a chimera-checked 16S rRNA gene database and workbench compatible with ARB. Appl Environ Microbiol 2006, 72(7):5069-5072

13. Kibbe WA: OligoCalc: an online oligonucleotide properties calculator. Nucleic Acids Res 2007, 35(Web Server issue):W43-W46.

14. Morgulis A, Coulouris G, Raytselis Y, Madden TL, Agarwala R, Schaffer AA: Database indexing for production MegaBLAST searches. Bioinformatics 2008, 24(16):1757-1764.

15. Nadkarni MA, Martin FE, Jacques NA, Hunter N: Determination of bacterial load by real-time PCR using a broad-range (universal) probe and primers set. Microbiology 2002, 148(Pt 1):257-266.

16. Tamura K, Dudley J, Nei M, Kumar S: MEGA4: Molecular Evolutionary Genetics Analysis (MEGA) software version 4.0. Mol Biol Evol 2007, 24(8):1596-1599.

17. DJLane1991Nucleic acid techniques in bacterial systematicsJohn Wiley and SonsNew York, NYLane DJ: Nucleic acid techniques in bacterial systematics. New York, NY: John Wiley and Sons; 1991.

18. Jari Oksanen, F. Guillaume Blanchet, Roeland Kindt, Pierre Legendre, Peter R. Minchin, R. B. O'Hara, Gavin L. Simpson, Peter Solymos, M. Henry, H. Stevens, Helene Wagner (2011). vegan: Community Ecology Package. R package version 2.0-2. Accessed at: [http://CRAN.R-project.org/package=vegan]

19. RDCTeam2008R: A language and environment for statistical computingR Foundation for Statistical ComputingViennaTeam RDC: R: A language and environment for statistical computing. Vienna: R Foundation for Statistical Computing; 2008.

20. Lee ZM, Bussema C 3rd, Schmidt TM: rrnDB: documenting the number of rRNA and tRNA genes in bacteria and archaea. Nucleic Acids Res 2009, 37 (Database issue):D489-D493.

21. Gaubatz J, Prashad N, Cutler RG: Ribosomal RNA gene dosage as a function of tissue and age for mouse and human. Biochim Biophys Acta 1976, 418(3):358-375.
22. Consortium IHGS: Finishing the euchromatic sequence of the human genome. Nature 2004, 431(7011):931-945

23. Dolezel J, Bartos J, Voglmayr H, Greilhuber J: Nuclear DNA content and genome size of trout and human. Cytometry A 2003, 51(2):127-128. author reply 129 .

24. Bottger EC: Frequent contamination of Taq polymerase with DNA. Clin Chem 1990, 36(6):1258-1259.

25. Dreier J, Stormer M, Kleesiek K: Two novel real-time reverse transcriptase PCR assays for rapid detection of bacterial contamination in platelet concentrates. J Clin Microbiol 2004, 42(10):4759-4764.

26. Klaschik S, Lehmann LE, Raadts A, Hoeft A, Stuber F: Comparison of different decontamination methods for reagents to detect low concentrations of bacterial 16 S DNA by real-time-PCR. Mol Biotechnol 2002, 22(3):231-242.

27. Meier A, Persing DH, Finken M, Bottger EC: Elimination of contaminating DNA within polymerase chain reaction reagents: implications for a general approach to detection of uncultured pathogens. J Clin Microbiol 1993, 31(3):646-652.

28. Rand $\mathrm{KH}$, Houck H: Taq polymerase contains bacterial DNA of unknown origin. Mol Cell Probes 1990, 4(6):445-450.

29. Klappenbach JA, Dunbar JM, Schmidt TM: rRNA operon copy number reflects ecological strategies of bacteria. Appl Environ Microbiol 2000, 66 (4):1328-1333.

30. Stevenson BS, Schmidt TM: Life history implications of rRNA gene copy number in Escherichia coli. Appl Environ Microbiol 2004, 70(11):6670-6677.

31. Morales SE, Holben WE: Empirical testing of $16 \mathrm{~S}$ rRNA gene PCR primer pairs reveals variance in target specificity and efficacy not suggested by in silico analysis. App/ Environ Microbiol 2009, 75(9):2677-2683.

32. Ludwig W, Schleifer KH: How quantitative is quantitative PCR with respect to cell counts? Syst App/ Microbio/ 2000, 23(4):556-562.

33. Hughes MS, Beck LA, Skuce RA: Identification and elimination of DNA sequences in Taq DNA polymerase. J Clin Microbiol 1994, 32(8):2007-2008.

34. Corless CE, Guiver M, Borrow R, Edwards-Jones V, Kaczmarski EB, Fox AJ: Contamination and sensitivity issues with a real-time universal $16 \mathrm{~S}$ rRNA PCR. J Clin Microbiol 2000, 38(5):1747-1752.

35. Sarkar G, Sommer SS: Removal of DNA contamination in polymerase chain reaction reagents by ultraviolet irradiation. Methods Enzymol 1993, 218:381-388.

36. Silkie SS, Tolcher MP, Nelson KL: Reagent decontamination to eliminate false-positives in Escherichia coli qPCR. J Microbiol Methods 2008, 72 (3):275-282.

doi:10.1186/1471-2180-12-56

Cite this article as: Liu et al.: BactQuant: An enhanced broad-coverage bacterial quantitative real-time PCR assay. BMC Microbiology 2012 12:56.

\section{Submit your next manuscript to BioMed Central and take full advantage of:}

- Convenient online submission

- Thorough peer review

- No space constraints or color figure charges

- Immediate publication on acceptance

- Inclusion in PubMed, CAS, Scopus and Google Scholar

- Research which is freely available for redistribution 